\title{
Multidimensional indices and orders of diversity
}

\author{
K. Mosler
}

Universität zu Köln, Albertus-Magnus-Platz, 50923 Köln, Germany.E-mail: mosler@wiso.uni-koeln.de

Keywords: Concentration order, Gini-Simpson index, Majorization, Multivariate diversity and evenness indices.

Abstract: This paper presents several indices to describe multivariate diversity and evenness. Multivariate generalizations of the Gini-Simpson index and the Rosenbluth index are proposed to measure diversity. A multivariate Gini ratio is also presented to measure evenness. These indices extend the usual univariate measures; they reflect not only the diversity of marginal distributions but also the dependence structure of abundance. The indices fulfill desirable measurement properties and are consistent with certain orderings of multivariate distributions. An order of concentration surfaces and a majorization order are also surveyed shortly.

\section{Introduction}

Consider a community of species living in a space that has several dimensions. The dimensions may relate to limited resources or to attributes of importance. For example, resource dimensions may be light and humidity for plants or prey and space for animals. Examples of importance dimensions are population density (or total number) and species biomass for plants as well as for animals. Our aim is to measure the $\alpha$-diversity of the given community and to compare it with the diversities of other communities. The measures - indices and orders - shall be based not only on one dimension but on several dimensions simultaneously and they shall also reflect possible interactions between the dimensions.

There exists a classical literature on the univariate measurement of diversity; see e.g., the comprehensive and unified treatments by Pielou (1977), Solomon (1979), Rao (1982), Patil and Taillie (1982) and many recent, more special studies. Tóthmérész (1995) surveys and discusses twelve univariate methods of diversity ordering. However, there exist rather few approaches to multivariate measurement, e.g., Alatalo and Alatalo (1977) and Loehle and Wein (1994).

Section 2 treats the univariate measurement. Several popular diversity indices, the concentration curve and concentration order, two evenness indices, and the Lorenz order are surveyed. In Section 3, the multivariate GiniSimpson index is introduced. Section 4 presents a multivariate majorization, called relative dilation, and a multivariate concentration order together with monotonicity properties of indices including a multivariate Rosenbluth diversity index. Section 5 discusses a multivariate Gini ratio and Section 6 concludes the paper.

\section{Univariate indices and orders}

If just one resource or one importance value is relevant, a univariate measurement problem has to be solved. Many indices have been proposed and used in the literature to measure the diversity of a given community in a one-dimensioned space. Let the community consist of $S$ species and each of them use a share $p_{i}$ of the limited resource (or bear relative importance $p_{i}$ ), $i=1,2, \ldots, S$.

\subsection{Indices of diversity}

The following diversity indices have been widely used in ecology,

- Number of species $S$,

- Shannon-Wiener index

$D_{1}=-\sum_{i=1}^{S} p_{i} \log p_{i}$

- Gini-Simpson index

$D_{2}=-\log \sum_{i=1}^{S} p_{i}^{2}$

- Rényi's $\alpha$-entropy

$D_{\alpha}=\frac{1}{1-\alpha} \log \sum_{i=1}^{S} p_{i}^{\alpha}, \alpha>0$, 
where $\log y$ denotes the natural logarithm of $y$. The family $D_{\alpha}$ includes the Gini-Simpson index and, in the limit $\alpha \rightarrow$ 1 , also the Shannon-Wiener index. Rényi's $D_{\alpha}$ is frequently used in the following variant, which is mentioned as the

- Hill index

$H_{\alpha}=\exp \left(D_{\alpha}\right)=\left(\sum_{i=1}^{S} p_{i}^{\alpha}\right)^{\frac{1}{1-\alpha}}, \alpha>0$.

To measure concentration, which is the opposite of diversity, the Rosenbluth concentration index $A$ is well known in economics,

$A=\frac{1}{S(1-R)}$,

where

$R=\frac{1}{S} \sum_{i=1}^{S}(2 i-S-1) p_{(i)}$

and $p_{(i)}$ is the $i$-th share ordered from below, $p_{(1)} \leq p_{(2)} \leq$ $\ldots \leq p_{(S)}$. To measure diversity, we introduce the

- Rosenbluth diversity index

$B=\frac{1-A}{1+A}=\frac{S(1-R)-1}{S(1-R)+1}$.

All these diversity indices measure which shares of the resource are used by how many species.

\subsection{Concentration order}

The distribution of resources among species is nicely described by a graphical device, the concentration curve (which is also popular in economics):

- Concentration rates:

$C_{i}=p_{(S)}+\ldots+p_{(S-i+1)}, i=1,2, \ldots, S$,

and draw the points

$(0,0),\left(C_{1}, 1\right), \ldots,\left(C_{S-1}, S-1\right),(1, S)$

in two-space and connect them by a piecewise linear curve. This curve is the concentration curve. For each $i$, the $i$-th concentration rate $C_{i}$ equals the total share of the resource held by the $i$ "largest" species; its negative, $-C_{i}$, serves as another index of diversity. The area $G$ below the concentration curve is related to the Rosenbluth diversity index $B$, there holds $B=(2 G-1) /(2 G+1)$.
When two communities $P$ and $Q$ are compared, the first having $S$ species and shares $p_{1}, \ldots p_{S}$ and the second having $T$ species and shares $q_{1}, \ldots q_{T}$, both concentration curves may be drawn. If the concentration curve of the second lies below that of the first, we say that $Q$ majorizes $P$ in concentration order. This is tantamount saying that all concentration rates are larger with $Q$ than with $P$; the second community is more concentrated, that is less $d i$ verse than the first. Obviously, the concentration order implies that $S \geq T$. Actually, it implies much more, namely:

Proposition 1: If one of two communities is less diverse than the other in the concentration order then each of the above mentioned diversity indices has a smaller value for the first community than for the second.

The proposition is known. For $A$ it follows from the fact that $B$ is an increasing function of the area below the concentration curve. For $D_{\alpha}$ and, hence, for $H_{\alpha}$ it follows from standard results on majorization (Marshall and Olkin 1979, Tong 1983, Mosler 1994).

As the concentration order has an obvious interpretation in terms of diversity and in view of Proposition 1, it seems rather natural to postulate for any univariate index of diversity that it should decrease with the concentration order.

\subsection{Indices of evenness}

The measurement of evenness abstracts from the number of species present in the community but focusses on their relative importance. It quantifies the slope of the importance value sequence and measures its flatness (Whittaker 1972, pp. 217 ff).

The question is here which shares of the resource are used by which parts of the species. Univariate indices that measure non-evenness are

- Gini's ratio

$R=\frac{1}{S} \sum_{i=1}^{S}(2 i-S-1) p_{(i)}$,

- Coefficient of variation

$v=\sqrt{S \sum_{i=1}^{S} p_{i}^{2}-1}$

The coefficient of variation equals the standard deviation of the $p_{i}$ divided by their mean. Indices of evenness are obtained by going either to the negative or the reciprocal of $R$ and $v$. 
Both the Gini ratio $R$ and the coefficient of variation $v$ are closely related with diversity indices, $R$ with the Rosenbluth diversity index $B$, and $v$ with the Gini-Simpson index $D_{2}$,

$$
\begin{aligned}
& D_{2}=-\log \frac{v^{2}+1}{S}, \\
& B=\frac{S(1-R)-1}{S(1-R)+1} .
\end{aligned}
$$

Equation (1) will be employed in the sequel to define a proper multivariate extension of index $D_{2}$.

The relevant partial order in the univariate measurement of evenness is the Lorenz order, which is closely related to the concentration order. The piecewise linear connection of the points

$(0,0),\left(\frac{1}{S}, 1-C_{S-1}\right),\left(\frac{2}{S}, 1-C_{S-2}\right), \ldots,\left(\frac{S-1}{S}, 1-C_{1}\right),(1,1)$

in two-space is the Lorenz curve of $P$ with shares $p_{1}, \ldots, p_{S}$. If the Lorenz curve of a community $Q$ lies below that of a community $P$, then $Q$ is said to majorize $P$ in Lorenz order. Note that in case $S=T$ this is equivalent to majorizing in the concentration order.

A natural postulate for any index of evenness is that it should decrease with the Lorenz order. As the usual Gini ratio equals twice the area between the Lorenz curve and the diagonal of the unit square, the negative (or reciprocal) of the Gini ratio fulfills that postulate. The same does the negative (or reciprocal) of the coefficient of variation.

\section{A multivariate Gini-Simpson index}

Now, we turn to the problem of measuring diversity when there is more than one dimension of resources or importance variables. Assume there are $S$ species and $J$ resources and let $p_{i j}$ denote the share of resource $j$ that is used by species $i, S \sum_{i=1}^{S} p_{i j}=1$ for all $j$. Assume that every species uses at least one resource, that is, $\min _{j} p_{i j}>$ 0 for every $i$. The shares of each species are collected in a vector $\mathbf{p}_{i}=\left(p_{i 1}, \ldots, p_{i J}\right), i=1, \ldots, S$.

I propose a multivariate Gini-Simpson index that is based on a multivariate standardized variance as follows. Define, for $j, k=1, \ldots, J$,

$\sigma_{j k}^{*}=S \sum_{i=1}^{S} p_{i j} p_{i k}-1$

which corresponds to the covariance between the resources $j$ and $k$, and let $\Sigma^{*}=\left[\sigma_{j k}^{*}\right]$ be the respective $J \times$
$J$ matrix. More precisely, $\sigma_{j k}^{*}$ is $S^{2}$ times the covariance between the vectors of shares which belong to resources $j$ and $k$. The $J$-variate Gini-Simpson index is defined by (1) by substituting $v=\sqrt{\operatorname{det} \Sigma^{*}}$, that is

$D_{2}=-\log \frac{\operatorname{det} \sum^{*}+1}{S}$,

and the corresponding Hill index is obtained as

$$
H_{2}=e^{D_{2}}=\frac{S}{\operatorname{det} \sum^{*}+1}
$$

Several properties are desirable postulates with any index of diversity or evenness:

- Anonymity: The index does not change if the species are renamed.

- Scale invariance: The index does not change if the scale of measurement is changed in one or several attributes.

- Continuity: The index is continuous in all its arguments.

Proposition 2: The multivariate Gini-Simpson index $\mathrm{D}_{2}$ and the multivariate Hill index $\mathrm{H}_{2}$ fulfill the postulates of anonymity, scale invariance, and continuity.

The proposition is obvious. The ranges of the indices are

$$
] \log \frac{S}{J(S-1)+1}, \log S\left[\text { for } D_{2}\right.
$$

$$
] \frac{S}{J(S-1)+1}, S\left[\text { for } \mathrm{H}_{2},\right.
$$

where ]. , . [ denotes the open interval. Further, any index of diversity or evenness is supposed to increase with socalled regressive transfers. In the next section, I introduce a multivariate version of such transfers and state that $D_{2}$ and $\mathrm{H}_{2}$ increase with them.

\section{Multivariate orders of diversity and evenness}

We first define a majorization order, relative dilation, for comparisons of evenness. This order is also relevant to diversity comparisons when the number of species does not change. Afterwards, we define a concentration order 
for comparisons of diversity when the numbers of species are different.

\subsection{Multivariate dilations}

Consider two communities $P$ and $Q$ which are characterized by their sequences of share vectors $\mathbf{p}_{1}, \ldots, \mathbf{p}_{S}$ and $\mathbf{q}_{1}, \ldots, \mathbf{q}_{T}$, respectively. Note that the number of species, $S$, in $P$ may differ from the number of species, $T$, in $Q$. Community $Q$ is said to be a relative dilation of $P$ if there exist numbers $\beta_{i k} \geq 0$ such that

$\mathbf{p}_{i}=\frac{T}{S} \sum_{k=1}^{T} \beta_{i k} \mathbf{q}_{i k}, \sum_{k=1}^{T} \beta_{i k}=1, \sum_{i=1}^{S} \beta_{i k}=\frac{S}{T}$.

For example, let

$\mathbf{p}_{1}=(0.6,0.48), \mathbf{p}_{2}=(0.4,0.52)$, and

$\mathbf{q}_{1}=(0.3,0.2), \mathbf{q}_{2}=(0.5,0.2), \mathbf{q}_{3}=(0.1,0.4)$,

$\mathbf{q}_{4}=(0.1,0.2)$.

Then, using

$\beta_{11}=0.0, \beta_{12}=0.5, \beta_{13}=0.2, \beta_{14}=0.3$,

$\beta_{21}=0.5, \beta_{22}=0.0, \beta_{23}=0.3, \beta_{24}=0.2$,

we see that $Q$ is a relative dilation of $P$.

Relative dilation may be interpreted in a dynamic context as follows: Community $Q$ changes to community $P$ while each species $i$ in $P$ takes part $(T / S) \beta_{i k}$ of the resources from each species $k$ in $Q$. As a simple special case, let $S=T$ and assume that all species do not change resources except two species, say $i=1$ and 2 . This means that $\beta_{i k}=0$ if $i, k \notin\{1,2\}$. In this case species 1 obtains some part $\beta_{12}$ of the other species' resource vector and retains part $\beta_{11}$ of its own, and so does species 2 vice versa. The special case is called a simple regressive transfer from $Q$ to $P$. Any relative dilation with $S=T$ can be split into a reverse series of simple regressive transfers, each from one single species to another. A general relative dilation with $S \neq T$ differs from the previous by a rescaling, which multiplies the $Q$-shares with the factor $T / S$. For details, see Koshevoy and Mosler (1997).

Relative dilation induces a partial order between communities. When there is only one resource, $J=1$, this order is equivalent to the usual Lorenz order of $T \cdot \mathbf{q}_{1}, \ldots, T \cdot \mathbf{q}_{T}$ over $S \cdot \mathbf{p}_{1}, \ldots, S \cdot \mathbf{p}_{S}$. Multivariate relative dilation implies relative dilation in every single dimension.

Increasingness with respect to a simple regressive transfer is a postulate for any index of evenness as well as diversity:
- Regressive Transfer: The index increases if two species exchange parts of their resources.

Proposition 3: The multivariate Gini-Simpson index decreases with relative dilations. In particular, it satisfies the postulate of regressive transfer.

The proposition is proved by standard majorization arguments (e.g., Mosler 1994).

\subsection{Multivariate concentration order}

To introduce multivariate versions of the concentration curve and the concentration order, we first define a concentration function $c(z)$ with argument $z$ in the $J$-variate cube,

$$
c(z)=\min \sum_{i=1}^{S} \theta_{i}, \quad z \in[0,1]^{J}
$$

where the minimum is taken over all $\theta_{1}, \ldots, \theta_{S}$ with

$$
\sum_{i=1}^{S} \theta_{i} p_{i j} \geq z_{j} \quad \text { for all } j .
$$

The graph of $c(z)$ is a set in $(J+1)$-space. If $J=2$, then it is named the concentration surface, in larger dimensions the concentration hypersurface.

Let us explain the concentration hypersurface. For a given vector of resource shares, $z \in[0,1]^{J}$, determines the minimum size of an aggregate species which uses these shares or more. The aggregate species consists of portions $\theta_{i}$ of each species $i$, and the sum of the portions is the size of the aggregate species. Its minimum size defines the value of the $J$-variate concentration function at $z$. Note that in case $J=1$ the concentration hypersurface is just the usual concentration curve (Figure 1).

To compare two communities with respect to their diversity, their concentration functions may be employed. If one concentration function is smaller than the other at every $\operatorname{argument} z$, the concentration hypersurfaces are ordered. We call this the multivariate concentration order.

It can be proved that relative dilation with equal species number implies concentration order: If $S=T$ and $Q$ is a relative dilation of $P$ then the concentration function of $Q$ is smaller than that of $P$. Moreover, it can be shown that index $D_{2}$ decreases with the concentration order:

Proposition 4: If $S \geq T$ and the concentration hypersurface of $Q$ is below that of $P$, then the Gini-Simpson index $D_{2}$ of $Q$ is smaller than that of $P$. 


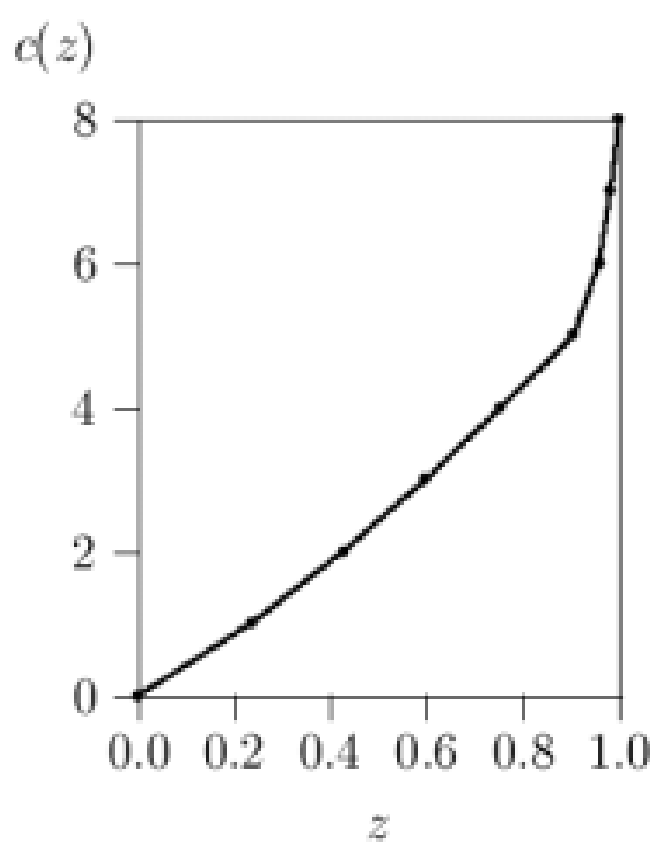

Figure 1. The concentration curve for one dimension.

\subsection{A numerical example}

Consider a community of $S=8$ species with shares in two resource dimensions. Let the transpose of the $8 \times 2$ matrix of shares be given by

$$
\left[p_{i j}\right]^{\prime}=\left[\begin{array}{llllllll}
.0179 & .1519 & .0521 & .1715 & .1563 & .2348 & .1915 & .0238 \\
.0065 & .0877 & .0214 & .2809 & .0715 & .3155 & .1781 & .0384
\end{array}\right]
$$

Figure 1 exhibits the concentration curve of the first dimension alone, and Figure 2 the concentration surface of both dimensions. The figures are reproduced from Koshevoy and Mosler (1999). Curves like these may also be mentioned as diversity profiles; the term was proposed by Patil and Taillie (1979).

\subsection{A multivariate Rosenbluth diversity index}

As in the univariate case the Rosenbluth diversity index is determined by the area below the concentration curve, in dimension $J \geq 2$, the same can be done with the concentration hypersurface. Let $G$ denote the volume below the concentration hypersurface. A multivariate Rosenbluth diversity index is defined by

$$
B=\frac{G-\frac{J}{J+1}}{G+\frac{J}{J+1}}
$$

Concerning diversity orderings there holds:

Proposition 5:

(i) The multivariate Rosenbluth diversity index $B$ satisfies the postulates of anonymity, scale invariance, and continuity. There holds always $B \geq 0$. The maximum $B=$ $(S-1) /(S+1)$ is achieved if and only if all share vectors are identical, that is $\mathbf{p}_{i}=(1 / S, \ldots, 1 / S)$ for all species $i$.

(ii) $B$ decreases with the multivariate concentration order and, if $S=T$, also with the relative dilation order.

Part (i) and monotonicity in the concentration order are immediately seen from the definition. Many other properties of the multivariate concentration order are found in Koshevoy and Mosler (1999).

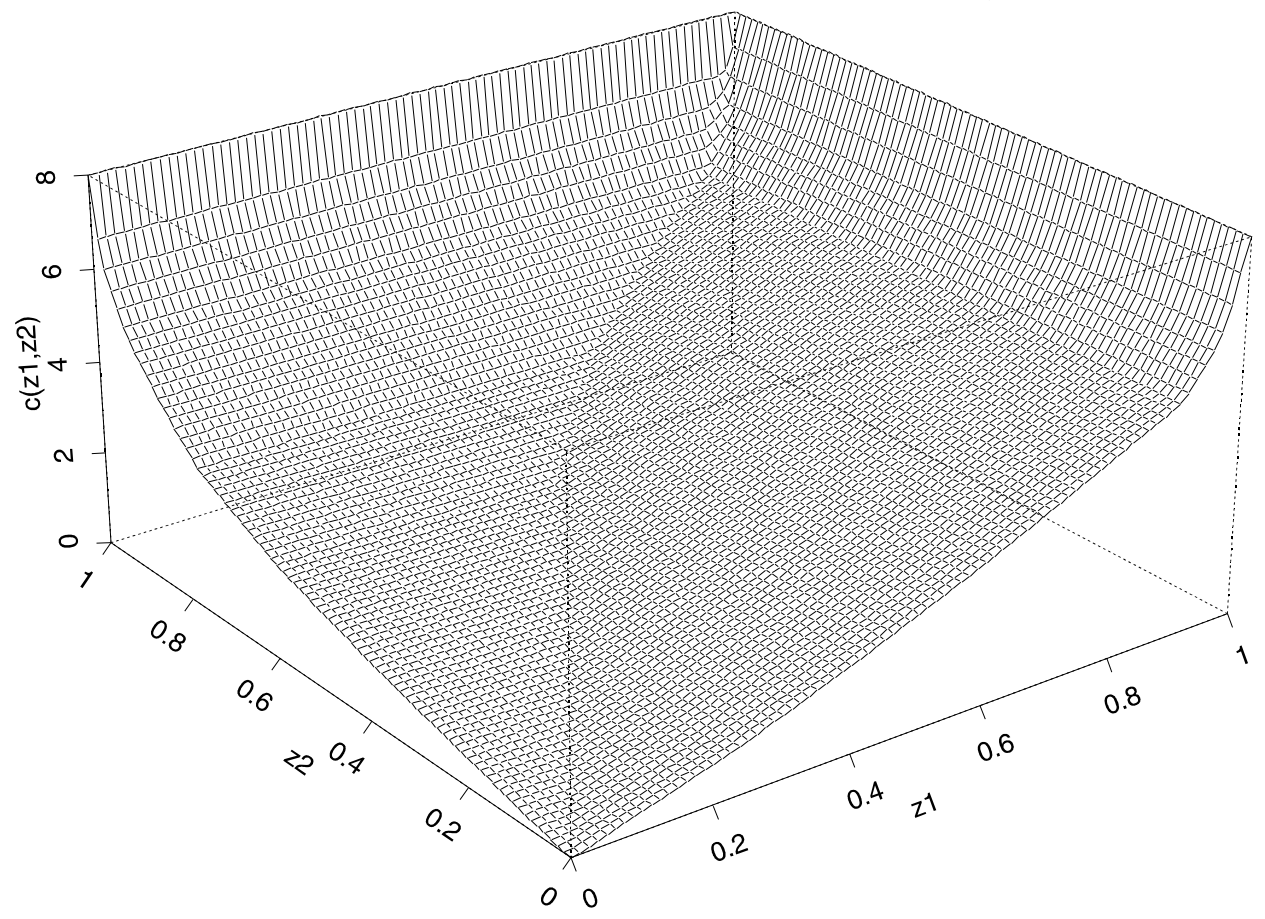

Figure 2. The concentration hypersurface for two dimensions. 


\section{A multivariate Gini ratio}

To measure evenness in more than one dimension,various extensions of the usual Gini ratio are possible. Instead of defining a multivariate Gini ratio through Equation (2) and the above multivariate Rosenbluth index, I introduce a notion which is easier to handle.

The multivariate Gini ratio is given by

$$
R=\frac{1}{2 S^{2} J} \sum_{i=1}^{S} \sum_{k=1}^{S}\left\|\mathbf{p}_{i}-\mathbf{p}_{k}\right\|,
$$

with

$$
\left\|\mathbf{p}_{i}-\mathbf{p}_{k}\right\|=\left(\sum_{j=1}^{J}\left(p_{i j}-p_{k j}\right)^{2}\right)^{1 / 2}
$$

being the Euclidean distance. Note that $R$ reduces to the usual Gini ratio when $J=1$.

\section{Proposition 6:}

(i) The multivariate Gini ratio $R$ satisfies the postulates of anonymity, scale invariance and continuity. There holds always $R \geq 0$. The minimum $R=0$ is achieved if and only if all share vectors are identical.

(ii) $R$ has the following ceteris paribus property: If we add a $(J+1)$-th dimension in which the species do not differ, the Gini ratio changes by a factor $J /(J+1)$ only.

(iii) $R$ is strictly increasing with relative dilation.

Thus, as an index of evenness, the negative (or the reciprocal) of the multivariate Gini ratio increases with the reverse of a relative dilation. Part (i) of the proposition is immediate from the definition; for (ii) and (iii) see Koshevoy and Mosler (1997)

The question remains in which respect an index of evenness like $R$ differs from an index of diversity like $D_{2}$ or $B$. Consider the following postulates:

- Replication invariance: The index remains unchanged if the community is replicated to $k \geq 2$ similar communities, that is, if each species $i$, which holds a vector $\left(p_{i 1}, \ldots, p_{i J}\right)$ of resource shares, is substituted by $k$ different species each holding a vector $\left(p_{i 1} / k, \ldots, p_{i j} / k\right)$.

- Replication monotonicity: The index increases strictly if the community is replicated to $k \geq 2$ similar communitities.

- Cardinal replication monotonicity: The index increases by a factor $k$ if the community is replicated to $k \geq 2$ similar communities.

Proposition 7:

(i) R satisfies the postulate of replication invariance. (ii) $\mathrm{D}_{2}, \mathrm{H}_{2}$ and $\mathrm{B}$ satisfy the postulate of replication monotonicity.

(iii) $\mathrm{H}_{2}$ satisfies also the postulate of cardinal replication monotonicity, but $D_{2}$ and $B$ do not.

The proposition is easily verified from the definitions. Thus, the principal difference between diversity and evenness is that a diversity index increases under replication, while an evenness index remains constant.

\section{Concluding remarks}

To compare the diversity and evenness of communities, I have presented several indices and orderings and discussed their relations. Also, I introduced a new diversity index, the multivariate Gini-Simpson index, and investigated some of its properties.

Throughout the paper, diversity and evenness have been compared at given points in time or space. All measures presented here are local in the sense that no resource gradients and no allocation of species in some niche hyperspace are considered. They are also static in the sense that no evolution of diversity over time is modelled.

Several variants of the above multivariate indices make sense. Other Gini-Simpson indices are obtained by plugging other notions of multivariate variance into Equation (1). In particular, the trace of the matrix $\Sigma^{*}$ may be used in place of the determinant; then a multivariate Gini-Simpson index, $\widetilde{D}_{2}$, is obtained which neglects possible interactions between the dimensions. Note that a properly transformed index, $\exp \left(\widetilde{D}_{2}\right)$, can be additively decomposed into the diversities of single dimensions.

Also, there are different ways to extend the Gini ratio to several dimensions. As the univariate Gini ratio equals twice the area between the Lorenz curve in two-space, a $J$-variate Gini ratio has been defined as the volume of a certain convex body in $(J+1)$-space (Mosler 1994, Koshevoy and Mosler 1997); this Gini ratio has similar properties as the above one but needs heavier computations. Also, distances other than the Euclidean may be employed in the multivariate Gini ratio, e.g., with different weights in the dimensions.

Multivariate entropy indices have been proposed and investigated by Maasoumi (1986).

Acknowledgements: I thank Béla Tóthmérész, János Izsák and Frédéric Gosselin for helpful remarks.

\section{References}

Alatalo, R. and R. Alatalo. 1977. Components of diversity: Multivariate analysis with interaction. Ecology 58: 900-906. 
Koshevoy, G. and K. Mosler. 1997. Multivariate Gini indices. J.Multivariate Analysis 60: 252-276.

Koshevoy, G. and K. Mosler. 1999. Measuring multidimensional concentration: A geometric approach. Allgemeines Statistisches Archiv 83: 173-189.

Loehle, C. and G. Wein. 1994. Landscape habitat diversity: A multiscale information theory approach. Ecol. Model. 73: 311-329.

Maasoumi, E. 1986. The measurement and decomposition of multidimensional inequality. Econometrica 54: 991-997.

Marshall, A.W. and I. Olkin. 1979. Inequalities: Theory of Majorization and its Applications. Academic Press, New York.

Mosler, K. 1994. Majorization in economic disparity measures. Linear Algebra and its Applications 199: 91-114.

Patil, G.P. and C. Taillie. 1982. Diversity as a concept and its measurement. JASA 77: 548-561.
Pielou, E.C. 1977. Mathematical Ecology. Wiley, New York.

Rao, C.R. 1982. Diversity and dissimilarity coefficients: A unified approach. Theoret. Popul. Biol. 21: 24-43.

Solomon, D.L. 1979. A comparative approach to species diversity. In: J.F. Grassle, G.P. Patil, W.K. Smith and C. Taillie (eds), Ecological Diversity in Theory and Practice. International Co-operative Publishing House, Fairland MD, pp 29-35.

Tong, Y.L. 1983. Some distribution properties of the sample speciesdiversity indices and their applications. Biometrics 39: 9991008.

Tóthmérész, B. 1995. Comparison of different methods for diversity ordering. J. Veg. Sci. 6: 283-290.

Whittaker, R.H. 1972. Evolution and measurement of species diversity. Taxon 21: 213-251. 